\title{
Mesons in ultra-intense magnetic field: an evaded collapse
}

\author{
B.O. Kerbikov ${ }^{1,2,3, a}$, M.A. Andreichikov ${ }^{1, b}$, and Yu.A. Simonov ${ }^{1, c}$ \\ ${ }^{1}$ A.I. Alikhanov Institute for Theoretical and Experimental Physics, Moscow 117218, Russia \\ ${ }^{2}$ Lebedev Physical Institute, Moscow 119991, Russia \\ ${ }^{3}$ Moscow Institute of Physics and Technology, Dolgoprudny 141700, Moscow Region, Russia
}

\begin{abstract}
Spectra of $q \bar{q}$ mesons are investigated in the framework of the Hamiltonian obtained from the relativistic path integral in external homogeneous magnetic field. The spectra of all 12 spin-isospin $s$-wave states generated by $\pi$ - and $\rho$-mesons with different spin projections, are studied analytically as functions of the field strength. Three types of behavior with characteristic splittings are found. The results are in agreement with recent lattice calculations.
\end{abstract}

\section{Introduction}

The interest to the behavior of quarks, hadrons and atoms in strong magnetic field (MF) has been very high during the last decade. The outbrake of the research activity in this field was inspired by the fact that MF up to $e B \sim \Lambda_{Q C D}^{2} \sim 10^{19} G^{1}$ is generated during the early stages of peripheral heavyion collisions at RHIC and LHC. The field about four orders of magnitudes less is anticipated to operate in magnetars. The immediate question is what happens to the mass and the wave function of a meson embedded in such a strong MF. The answer to this question has been searched for in various approaches (see [1] for a list of references) including lattice simulations. In the present work the problem is investigated in the framework of the relativistic path integral Hamiltonian (PIH) formalism [2-4]. For pion this method has to be supplemented by the elements of chiral dynamics [5]. The analytical results will be compared with the lattice calculations presented recently in [1]. Before getting involved with the details of calculations it makes sense to relate the MF strength to some characteristic physical parameter which defines the spectrum of quark-antiquark meson states. From the textbooks we know that for the hydrogen atom the critical, or the so-called "atomic field", is $B_{a}=\frac{\alpha^{2} m_{e}^{2}}{|e|}=2.35 \cdot 10^{9} G$. This value corresponds to the situation when the magnetic, or Landau radius $l_{B}=(|e| B)^{-1 / 2}$ is equal to the Bohr radius. The QCD coupling constant $\alpha_{s} \sim 1$, the meson radius at $e B=0$ is determined by the QCD string tension $\sigma \simeq(0.15-0.18) \mathrm{GeV}^{2}$ [4]. It is therefore natural to define for the hadron spectra the critical MF as $B_{\sigma}=\sigma /|e| \simeq 10^{19} G$ which yields $l_{B} \simeq 0.6 \mathrm{fm}$. This value is approximately equal or smaller than the typical hadron size.

\footnotetext{
ae-mail: borisk@itep.ru

be-mail: andreichicov@mail.ru

ce-mail: simonov@itep.ru

${ }^{1}$ We use the relativistic system of units $\hbar=c=1, e^{2}=4 \pi \alpha$. Then $1 \mathrm{GeV}^{2} \simeq 5.12 \cdot 10^{19} \mathrm{G}$
} 
The determination of the hadron spectrum in MF is not an easy task. The first problem is to separate the center-of-mass (c.m.) motion. For the neutral nonrelativistic system in MF this can be done (with some qualifications) making use of the Pseudomomentum [6-9]. This approach was extended to the relativistic sector within the PIH framework in [4]. For a charged meson Pseudomementum method is applicable only for an unphysical model of a meson with two equally charged quarks [4]. In this contribution we present the results on the meson spectrum in MF both within the Pseudomomentum approach and in a new analytical method of constituents separation (CS). It will be argued that its accuracy is within $15 \%$ for ultra-strong $\mathrm{MF}(e B \gg \sigma)$, and within $20 \%$ for $e B<\sigma$. The method allows to study neutral and charged mesons in the same way. The results for the neutral mesons will be obtained both in Pseudomomentum and CS approaches. In this way the accuracy of the CS will be tested.

The most important question we have to answer is whether the meson spectrum in MF is bounded from below. In other words, does the meson mass reaches zero value at some MF strength. We shall point out the two dynamical mechanisms that might have led to such a collapse and explain why this does not happen. The paper is organized as follows. In section 2 the relativistic Hamiltonian based on path integral Feynman-Fock-Schwinger representation is written down and the spectral problem is formulated. In section 3 we discuss the possible types of meson mass trajectories in MF. Section 4 contains the analysis of perturbative corrections and potential reasons for the collapse of meson state in MF. In section 5 we present the main results in comparison with lattice calculations.

\section{The relativistic Hamiltonian and the spectral problem}

To find the meson masses in MF we use the path integral Hamiltonian (PIH) method based on the Feynman-Fock-Schwinger representation [2-4]. It allows with the help of Wilson loop to treat the interaction of quarks with external Abelian and non-Abelian fields in a gauge-invariant way. As it was shown in $[2-4,10]$ the quark-antiquark spectral problem in MF in PIH formalism is reduced to the bound states problem for the relativistic Hamiltonian which includes all the non-perturbative dynamics

$$
H_{q \bar{q}}=\sum_{i=1}^{2} \frac{\left(\mathbf{p}_{i}^{2}-e_{i} \mathbf{A}_{i}\right)^{2}+m_{i}^{2}+\omega_{i}^{2}-e_{i}\left(\boldsymbol{\sigma}_{i} \mathbf{B}\right)}{2 \omega_{i}}+\sigma\left|\mathbf{r}_{1}-\mathbf{r}_{2}\right| .
$$

Here $\omega_{i}$ is the $i$-th quark dynamical mass, or the einbein variable [2-4]. The MF is convenient to take in the symmetric gauge $\mathbf{A}_{i}=\frac{1}{2}\left(\mathbf{B} \times \mathbf{r}_{i}\right)$ since this gauge allows to define the angular momentum projection of each quark as a quantum number. The next step is to perform minimization with respect to $\omega_{i}$ which yields the physical spectrum

$$
\begin{gathered}
H_{q \bar{q}} \psi_{n}=M_{n} \psi_{n}, \\
\frac{\partial M_{n}}{\partial \omega_{i}}=0 .
\end{gathered}
$$

The total meson mass is a sum of the non-perturbative (dynamical) one obtained from (1)-(3) and the first-order perturbative contributions

$$
M_{t o t}=M_{0}+\left\langle\psi_{0}\left|v_{\mathrm{oge}}\right| \psi_{0}\right\rangle+\left\langle a_{s s}\right\rangle\left(\sigma_{1} \sigma_{2}\right)+\delta M_{S E},
$$

where $V_{\text {oge }}$ is the one-gluon exchange potential, and $a_{s s}$ and $\Delta M_{S E}$ are the spin-spin and self energy contributions. 
For the neutral hadrons (mesons and baryons) the eigenvalue problem (2) admits the exact solution which is obtained by the separation of the c.m. motion. To this end the pseudomomentum operator is introduced [6-9]

$$
\hat{\mathbf{F}}=\sum_{i=1}^{2}\left[\mathbf{P}_{i}+\frac{1}{2} e_{i}\left(\mathbf{B} \times \mathbf{r}_{i}\right)\right] .
$$

In MF the pseudomomentum takes the role of the mechanical momentum, commutes with the Hamiltonian, and is therefore a constant of motion. Physically, $\mathbf{F}$ is conserved since it takes into account the Lorentz force acting on particles in MF.

In [1] we have proposed a more general approach which allows to investigate the mass spectra of both neutral and charged mesons. This is the constituent separation (CS) method. The c.m. position $\mathbf{r}_{0}$ is fixed at the origin and an effective string tension $\sigma_{i}$ is attributed to each quark

$$
\sigma\left|\mathbf{r}_{1}-\mathbf{r}_{2}\right| \rightarrow \sigma_{1}\left|\mathbf{r}_{1}-\mathbf{r}_{0}\right|+\sigma_{2}\left|\mathbf{r}_{2}-\mathbf{r}_{0}\right|
$$

In this picture quarks may be considered as quasi-independent of the non-perturbative part of the interaction.

\section{Meson trajectories in strong MF}

In strong MF $e B \gg \sigma$ it is convenient to use for the spin degrees of freedom in (1) a basis in which the operator $\mathbf{B}\left(\frac{e_{1}}{2 \omega_{1}} \sigma_{1}+\frac{e_{2}}{2 \omega_{2}} \sigma_{2}\right)$ is diagonal. The four vectors forming this basis are $|++\rangle,|--\rangle,|+-\rangle,|-+\rangle$. One can easily obtain three types of asymptotic meson trajectories at $e B \rightarrow \infty$. The character of the trajectory is determined by the signs of the quark charges and the spin directions. According to the terminology adopted in atomic physics the trajectory is called low-field seeking (LFS) if the energy decreases as the MF decreases. The state which at $e B \rightarrow \infty$ is MF-independent may be called zerofield seeking (ZFS). The types of asymptotic trajectories are

a) ZFS: $e_{1} \sigma_{1}^{z}>0, e_{2} \sigma_{2}^{z}>0: M_{Z F S}(e B \rightarrow \infty)=2 \sqrt{\sigma}$,

b) $L F S 1: e_{1} \sigma_{1}^{z}>0, e_{2} \sigma_{2}^{z}<0: M_{L F S 1}(e B \rightarrow \infty)=\sqrt{2 e_{1} B}+\sqrt{2 \sigma}$,

c) LFS $2: e_{1} \sigma_{1}^{z}<0, e_{2} \sigma_{2}^{z}<0: M_{L F S 2}(e B \rightarrow \infty)=\sqrt{2 e_{1} B}+\sqrt{2 e_{2} B}$,

The trajectory LFS2 exhibits stronger MF dependence than LFS1. From (7) it follows that the $\left(\pi^{+}, \rho^{+}\right)$ family which contains $u$ and $\bar{d}$ quarks is distributed among the three above classes in the following way: $\rho^{+}\left(s_{z}=1\right)$ belongs to ZFS, $\pi^{+}\left(s_{z}=0\right)$ and $\rho^{+}\left(s_{z}=0\right)$ belong to LFS1, $\rho^{+}\left(s_{z}=-1\right)$ rests in LFS2. The same situation up to the sign change holds for $\left(\pi^{-}, \rho^{-}\right)$. The states $\pi^{0}$ and $\rho^{0}\left(s_{z}=0\right)$ contain $u \bar{u}$ and $d \bar{d}$ components. The charges of $u$ and $d$ are different and this results in an additional double splitting.

\section{Perturbative corrections}

As it was shown in [10], the color Coulomb interaction in presence of the MF contains a potential danger of a collapse for ZFS states. This reminds the "fall-to-the-center" phenomenon in the hydrogen atom enbedded in strong MF. The one-gluon exchange matrix element $\left\langle\psi_{0}\left|V_{O G E}\right| \psi_{0}\right\rangle$ has a negative sign, its absolute value grows with $e B$, and as shown on Fig.1 "fall-to-the-center" takes place at $e B \sim 10 \mathrm{GeV}^{2}$. To prevent the collapse the screening by the quark loops at the lowest Landau level (LLL) was introduced in [10]. 


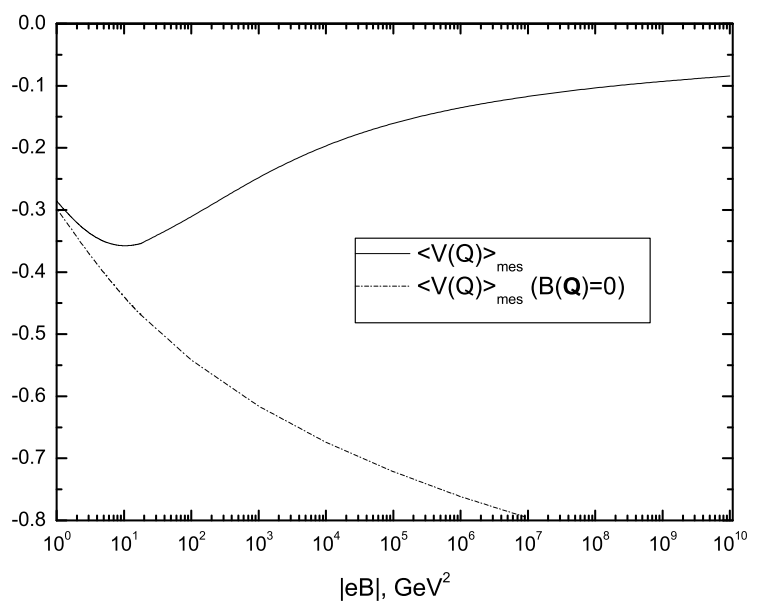

Figure 1. Color Coulomb matrix element $\left\langle\psi_{0}\left|V_{O G E}\right| \psi_{0}\right\rangle$ (in $\mathrm{GeV}$ ) in MF with screening (solid line) and without screening (dashed line) by $q \bar{q}$ pairs

As one can see from Fig.1, the color Coulomb collapse is really evaded. We remind that in superstrong MF radiative corrections screen the Coulomb potential in the hydrogen atom thus leading to the freezing of the ground state energy at the value $E_{0}=-1.7 \mathrm{KeV}[11,12]$. It is interesting to note that asymptotically at $e B \rightarrow \infty$ the matrix element $\left\langle\psi_{0}\left|V_{O G E}\right| \psi_{0}\right\rangle$ vanishes.

Another threat of a collapse comes from the hyperfine spin-spin interaction $\left\langle a_{S S}\right\rangle$. In the firstorder perturbation theory in PIH formalism it corresponds to the color-magnetic interaction of the form

$$
V_{S S}=\frac{8 \pi \alpha_{s}^{(0)}}{9 \omega_{1} \omega_{2}} \delta\left(\mathbf{r}_{1}-\mathbf{r}_{2}\right) \sigma_{1} \cdot \sigma_{2}
$$

In strong MF the ground state wave function acquires the form of an ellipsoid elongated in the direction of MF. At $e B \rightarrow \infty$ the transverse and longitudinal radii are $r_{\perp} \sim 1 / \sqrt{e B}, r_{z} \sim 1 / \sqrt{\sigma}$. This means that the focusing of the wave function at the origin and a divergent factor $|\psi(0)|^{2} \sim e B$ in the matrix element of $V_{S S}$. Not that the problem of singularity due to $\delta$-function interaction exists without MF as well. It is cured by smearing $\delta$-function $[13,14]$. In PIH formalism there is a natural cut-off parameter $\lambda \sim 1 \mathrm{GeV}^{-1}$. It corresponds to the correlation length of the stochastic vacuum gluonic field. The $\delta$-function is replaced by

$$
\delta(\mathbf{r}) \rightarrow \frac{1}{\pi^{3 / 2} \lambda^{3}} e^{-\frac{r^{2}}{\lambda^{2}}}
$$

In this way the "fall-to-the-center"is prevented for all ZFS states except for the $\pi^{0}$-meson. The $\pi^{0}$ trajectory is stabilized if one takes its chiral degrees of freedom into account. We also note that in [3] a general theorem was proven according to which the eigenvalues of the relativistic Hamiltonian in MF are positive. The explicit account of pion chiral dynamics [5] confirm this result. The main point is that GMOR relations remain valid for neutral pions in arbitrary-strong MF, while charged pions loose their chiral properties at $e B>\sigma$. 


\section{Results and conclusions}

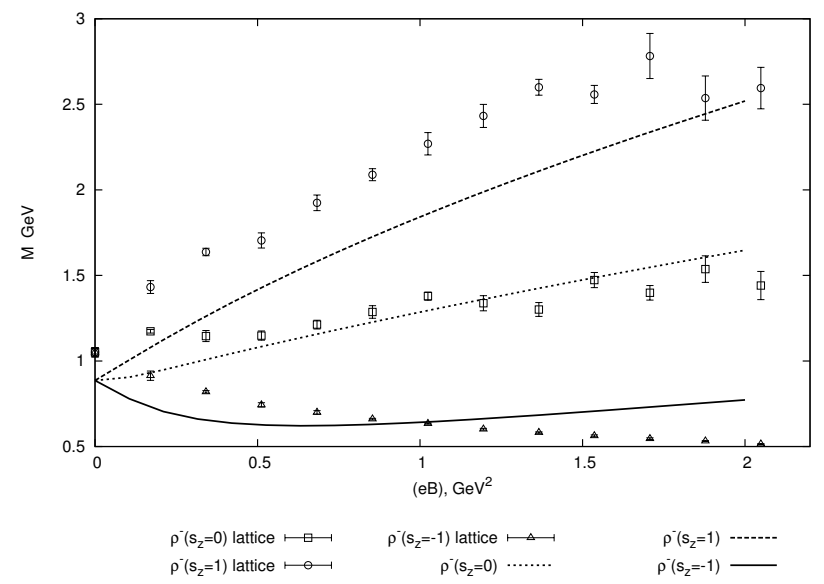

Figure 2. The $\rho^{-}$meson mass evolution in MF from our analytic and lattice data.

Below we present the results of our analytic calculations in comparison with the recent lattice results from [1]. In Fig.2 the $\rho^{-}$meson mass evolution in MF is shown.

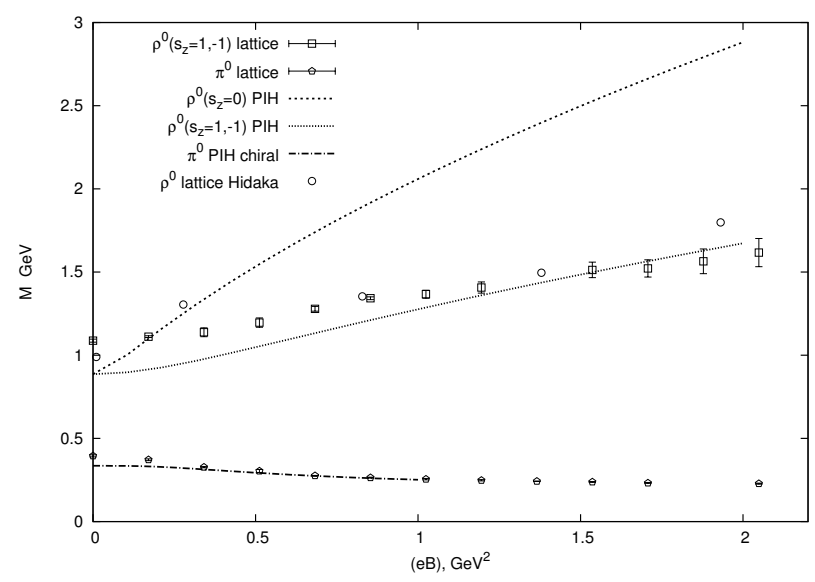

Figure 3. Mass evolution of $\left(\pi^{0}, \rho^{0}\right)(u \bar{u})$ family from analytic and lattice data (hollow circles are from [16]).

In Fig. 3 and Fig.4 results for $\pi^{0}$ and $\rho^{0}$ are exposed. One should keep in mind that $u \bar{u}$ and $d \bar{d}$ components give rise to their own trajectories. The growing trajectories belong to the LFS 2 class and the splitting is equal to $\sqrt{2}$. In Fig.5 we present the mass evolution of chiral and non-chiral $\pi^{-}$in comparison with the lattice data. The chiral effects provide the decrease of the mass to its physical value at $e B \rightarrow 0$.

In this work we have evaluated the trajectories of $\pi$ and $\rho$ meson masses as functions of the external MF. The meson quark content and pion chiral dynamics were thoroughly taken into account. The most 


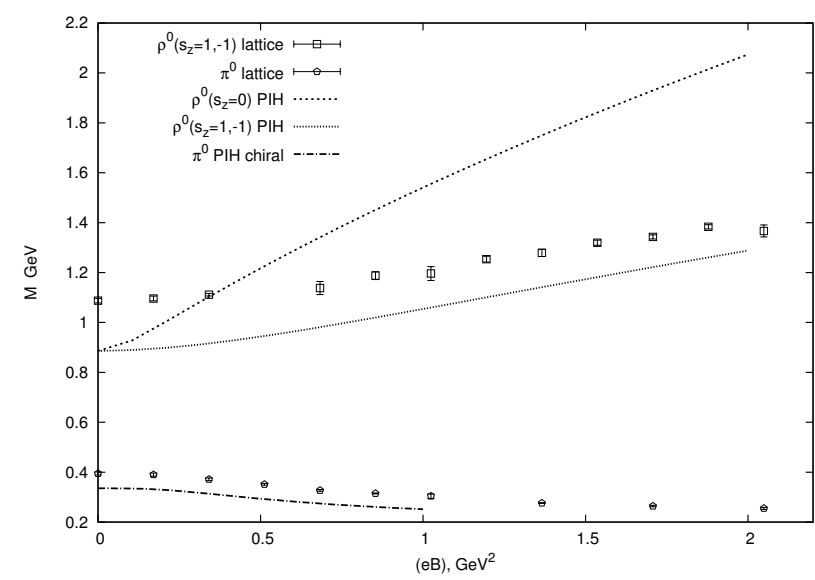

Figure 4. Mass evolution of $\left(\pi^{0}, \rho^{0}\right)(d \bar{d})$ family from analytic and lattice data

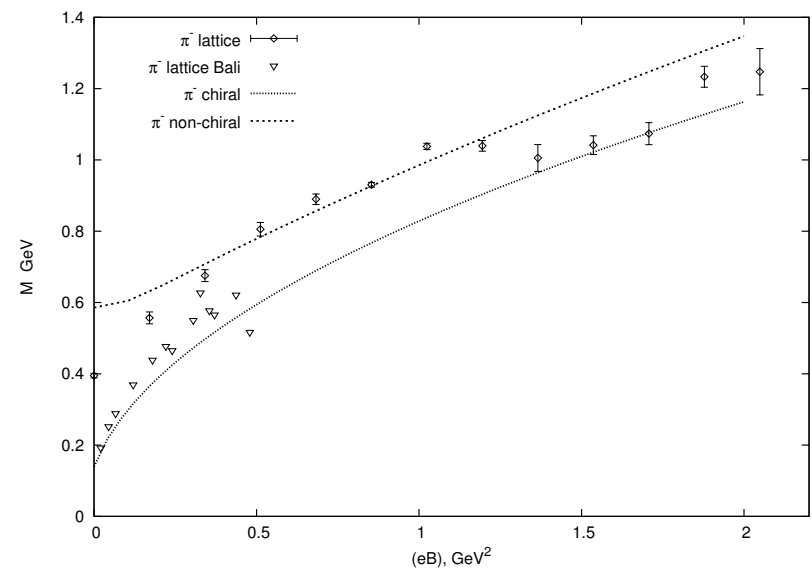

Figure 5. Mass evolution of the chiral (solid line) and non-chiral (dashed line) $\pi^{-}$meson in comparison with lattice data (triangles are from [15].

interesting problem was whether the mass remains finite in arbitrary strong MF. The collapse might have happened either due to color Coulomb interaction, or due to spin-spin potential proportional to $\delta$-function. We have shown that in both cases there are physical reasons why collapse is evaded. The analytic calculations of meson mass trajectories give the results which are in agreement with recent lattice simulations.

\section{Acknowledgement}

The authors are grateful to E.V.Luschevskaya and O.E.Solovjeva for kindly providings with their lattice results and to O.V.Teryaev for discussions. The authors are supported by the Russian Science Foundation grant number 16-12-10414. 


\section{References}

[1] M.A.Andreichikov, B.O.Kerbikov, E.V.Luschevskaya, Yu.A.Simonov, O.E.Solovjeva, arXiv:1610.06887 (2016).

[2] Yu.A.Simonov, Phys.Rev. D88, 025028 (2013), arXiv:1303.4952.

[3] Yu.A.Simonov, Phys.Rev. D88, 053004 (2013), arXiv:1304.0365.

[4] M.A.Andreichikov, B.O.Kerbikov, V.D.Orlovsky, Yu.A.Simonov, Phys.Rev. D87, 094029 (2013), arXiv:1304.2533.

[5] V.D.Orlovsky, Yu.A.Simonov, JHEP 1309, 135 (2013), arXiv:1306.2322.

[6] W.E.Lamb, Phys.Rev. 85, 59 (1952).

[7] L.P.Gor'kov, I.E.Dzyaloshinskii, Soviet Phys. JETP 26, 449 (1968).

[8] J.E.Avron, I.W.Herbst, B.Simon, Ann.Phys. (NY) 114, 431 (1978).

[9] H.Grotsch, R.A.Hegstrom, Phys.Rev. A4, 59 (1976).

[10] M.A.Andreichikov, V.D.Orlovsky, Yu.A.Simonov, Phys.Rev.Lett. 110, 162002 (2013).

[11] A.E.Shabad, V.V.Usov, Phys.Rev.Lett. 98180403 (2007).

[12] M.I.Vysotsky, S.I.Godunov, Phys.Uspekhi 57, 194 (2014).

[13] B.O.Kerbikov, M.I.Polikarpov, I.V.Shevchenko, Nucl.Phys. B331, 19 (1990).

[14] P.Brovetto, V.Maxia, M.Salis, Nuovo Cim. 14, 795 (1992).

[15] G.Bali, B.B.Brandt, G.Endrodi, B.Glassle, 33rd International Symposium on Lattice field theory, 14-18 Jul 2015, Kobe, Japan, arXiv:1510.03899.

[16] Y.Hidaka, A.Yamamoto, Phys.Rev. D87, 094502 (2013). 